\title{
Pharmacokinetic Prediction of Levofloxacin Accumulation in Tissue and Its Association to Tendinopathy
}

\author{
Loan Pham $^{1 *}$, John M. Christensen ${ }^{2}$, Rosita Rodriguez-Proteau ${ }^{2}$ \\ ${ }^{1}$ Camargo Pharmaceuticals, Cincinnati, USA; ${ }^{2}$ Department of Pharmaceutical Sciences, College of Pharmacy, Oregon State Univer- \\ sity, Corvallis, USA. \\ Email: "1pham@camargopharma.com,jmark.christensen@oregonstate.edu, Rosita.Proteau@oregonstate.edu
}

Received November $6^{\text {th }}, 2012$; revised December $20^{\text {th }}, 2012$; accepted January $10^{\text {th }}, 2013$

\begin{abstract}
Objectives: We investigated pharmacokinetic tissue distributions of Levofloxacin to explain adverse tendon incidents. Methods: The pharmacokinetic profiles of single and multiple dosing of $500 \mathrm{mg}$ Levofloxacin following oral and IV infusion administration were simulated. Monte Carlo simulation was used to simulate the drug concentration profiles in plasma and tissue after seven dosing regimens while varying the drug's elimination and distribution rates to analyze the effects of changing those rates on Levofloxacin accumulation in tissue. Results: Simulated data following oral and IV administration reflect well the reported data (mean simulated plasma Cmax $=6.59 \mu \mathrm{g} / \mathrm{mL}$ and $5.19 \mu \mathrm{g} / \mathrm{mL}$ for IV and oral versus $6.4 \mu \mathrm{g} / \mathrm{mL}$ and $5.2 \mu \mathrm{g} / \mathrm{mL}$ for observed clinical IV and oral route, respectively). Simulations of seven repetitive doses are also in agreement with reported values. Low elimination rates affect the drug concentration in plasma and tissue significantly with the concentration in plasma rising to $35 \mu \mathrm{g} / \mathrm{mL}$ at day 7 . Normal elimination rates together with escalation of distribution rates from plasma to tissue increase tissue concentration after 7 doses to $9.5 \mu \mathrm{g} / \mathrm{mL}$, a value is more than twice that of normal. Conclusions: Simulation can be used to evaluate drug concentration in different tissues. The unexpectedly high concentrations in some cases may explain the reason for tendinopathy in clinical settings.
\end{abstract}

Keywords: Monte Carlo Simulation; Tendon Incidents; Levofloxacin; Pharmacokinetic

\section{Introduction}

Tendinitis and tendon rupture have emerged with the popular use ofLevofloxacin [1-5]. Tendinopathy accounted for $4.1 \%$ of the cases and the compound could cause Achilles tendon rupture in $1 \%$ of subjects, a rate higher than previously thought $[6,7]$. The risk factors of Fluoroquinolones-induced tendinopathy include older age, concomitant corticosteroid therapy and renal dysfunction. Caution has been raised when prescribing a combination therapy of steroids and Levofloxacin to patients, particularly to those with known risk factors [8].

It has been shown that Levofloxacin levels were achievable in all tissue samples after a single intravenous dose despite high variability in its pharmacokinetics (PK) [9]. In bone and cartilage, Levofloxacin penetrated well into cortical and spongiosa tissue of femoral head and distal femur, with mean penetration ratios between 0.34 and 1.51. The penetration of Levofloxacin into bone was rapid, taking approximately 2 hours to reach the maximum concentration. By 5 hours, apparent equilibrium of Levofloxacin concentrations occurred between the bone tissues and plasma [9]. The Levofloxacin concentration

${ }^{*}$ Corresponding author. observed in plasma and in the interstitial space fluid (ISF) of lung tissue, and that of muscle and subcutaneous adipose tissue were significantly different after receiving a single intravenous dose of $500 \mathrm{mg}$ producing a 2-fold and 1.5-fold higher AUC from 0 to infinity (AUC0-inf) for the ISF of muscle and adipose tissue as compared to lung, respectively. The difference in AUC0-inf was postulated to be by higher clearance of Levofloxacin from lung tissue as compared to muscle and adipose tissue [10]. No difference in peak concentration between fat, skeletal muscle and lung was documented. Time to reach $\mathrm{C}_{\max }\left(\mathrm{T}_{\max }\right)$ values in adipose $(60 \mathrm{~min})$ and muscle tissue (80 $\mathrm{min})$ were shorter than that in lung tissue $(90 \mathrm{~min})$ yielding a shorter elimination half-life of Levofloxacin in the lung compared to muscle and adipose tissue [10]. The possible reason is that the capillary blood flow is higher in lung and much lower in peripheral soft tissue and possibly substantial differences in redistribution processes from tissue to the blood [11]. These results are of importance and should be taken into account when evaluating the distribution and clearance of Levofloxacin in different tissues regarding the relationship of capillary blood flow to the tissue and the concentration seen in peripheral blood and/or distribution and toxicity of the drug to 
a specific tissue and organ. It indicates that the distribution and peak concentration of Levofloxacin can be obtained in various tissues regardless of some limitation in blood perfusion and differences in $T_{\max }$ of the drug. In addition, stromatous tissue such as adipose tissue, articular capsule, trachea cartilage and tendon achieved similar concentrations of quinolones when subjected to a single dose of Fluoroquinolones intravenously in an experimental canine model albeit fat and the three latter kinds of tissues have significant discrepancy in structural constitution and distributive vasculatures [12]. Relatively, human Achilles tendon has little to no vasculature for itself [13] and is nurtured ultimately by permeation from the peripheral tissues suggesting that once a Fluoroquinolone has reached its peak concentration, it is likely to reside semi-permanently in tendon tissue and be harmful due to its prolonged residency. This may explain the higher prevalence of Achilles tendon incidents in diabetic and aged patients, who also suffer from a decreased circulatory network in Achilles tendon [13-17].

The accumulation of Levofloxacin/Fluoroquinolones in tendon may be the reason for tendon incidents, however there is no confirmed mechanism or relationship between plasma concentrations to tendonitis and Achilles tendon rupture or whether additional unknown reasons cause increased drug accumulation in tendon tissue leading to complications. Therefore, a simple process useful to determine drug distribution rate to tendon tissue and to predict the biological outcomes in order to provide early warning for Levofloxacin toxicity in daily practice is warranted. Monte Carlo simulation was used in this study to assess the usefulness of pharmacokinetic prediction in relation to Levofloxacin tissue accumulation and tendinopathy. The objective was to elucidate possible factors that will delineate the potential relationships between Levofloxacin accumulation in tendon tissue (such as Levofloxacin tissue concentration, and distribution processes) and tendinopathy incidents that will lead to further studies that will refine the understanding of the Mechanism that cause tendinopathy so that prevention of the events can be better predicted.

\section{Methods}

\subsection{Pharmacokinetics of Levofloxacin}

A two-compartment open model with first-order absorption and elimination process was used to describe Levo- floxacin plasma concentration time profile. The model is described by the following system of ordinary differential equations. Equation 1: $\mathrm{d} X_{a} / \mathrm{d} t=-K_{0} * X_{a}$. Equation (2): $\mathrm{d} X_{1} / \mathrm{d} t=X_{a} * K_{0}+X_{2} * K_{21}-X_{1} *\left(K_{12}+K_{e l}\right)$.

Equation (3): $\mathrm{d} X_{2} / \mathrm{d} t=X_{1} * K_{12}-X_{2} * K_{21}$. With $X_{\mathrm{a}}, X_{1}$, $X_{2}$ are the milligram amounts of drug in the gut, the central (plasma) and the peripheral compartments, respectively. $K_{\mathrm{a}}\left(\mathrm{hr}^{-1}\right)$ and $K_{0}(\mathrm{mg} / \mathrm{h})$ are the absorption rate constant and the intravenous (IV) infusion rate of Levofloxacin, respectively. $K_{e l}\left(\mathrm{~h}^{-1}\right)$ is the elimination rate constant from the central compartment. $K_{12}, K_{21}$ are the between-compartment transfer rate constants (all in $\mathrm{hr}^{-1}$ ).

The other pharmacokinetic parameters are the volume of distribution $(V)$. Equation (4): $C_{1}=X_{1} / V_{1}$. Equation (5): $C_{2}=X_{2} / V_{2}$. With $C_{1}, C_{2}$ are Levofloxacin concentration in $\mu \mathrm{g} / \mathrm{mL}$ in the central compartment and the peripheral compartments, respectively. $V_{1}, V_{2}$ represent volume of distribution in the central and peripheral compartments in $\mathrm{mL}$, respectively. $F$ is the fraction of dose absorbed. In extravascular models, the fraction of dose absorbed cannot be estimated separately. Therefore, $V_{1} / F$ was estimated together in PK modeling. Dpo is the administered dose orally. Pharmacokinetic parameter values (Table 1), obtained from literature [18], were used in Monte Carlo simulation of drug concentration versus time profiles for Levofloxacin after single or multiple oral and IV infusion dosing administration.

\subsection{Derivations of Drug Concentration in Plasma and Tissue Compartments after IV Infusion Single and Multiple Doses Using Laplace Transforms}

Recall the ordinary differential Equations (2) and (3) above:

$$
\begin{gathered}
\frac{\mathrm{d} X_{1}}{\mathrm{~d} t}=X_{0} K_{0}(u(t)-u(t-T))+X_{2} K_{21}-\left(K_{12}+K_{e l}\right) X_{1} \\
\frac{\mathrm{d} X_{2}}{\mathrm{~d} t}=X_{1} K_{12}-X_{2} K_{21}
\end{gathered}
$$

where: $u(t)$ is the unit function

$$
u(t)= \begin{cases}1, & t>0 \\ 0, & t \leq 0\end{cases}
$$

and $T$ is the duration time of infusion.

Using the Laplace transform, we have the following

Table 1. Summary Levofloxacin pharmacokinetics used in Monte Carlo Simulation Derivations of drug concentration in plasma and tissue compartments after IV infusion single and multiple doses using Laplace transforms.

\begin{tabular}{ccccccccc}
\hline & $K_{12}\left(\mathrm{~h}^{-1}\right)$ & $K_{21}\left(\mathrm{~h}^{-1}\right)$ & $V_{1}(\mathrm{~L})$ & $\mathrm{CL}(\mathrm{L} / \mathrm{h})$ & $V_{2}(\mathrm{~L})$ & $t_{1 / 2}(\mathrm{~h})$ & $K_{e l}$ & $\mathrm{~T}_{\max }$ \\
\cline { 2 - 8 } Mean & 0.487 & 0.647 & 18.92 & 9.27 & 51.05 & 7.4 & 0.099 & 1.3 \\
Median & 0.384 & 0.596 & & 9.01 & & 0.9 & & \\
SD & 0.378 & 0.391 & & 4.31 & & & & 0.5 \\
\hline
\end{tabular}


equation in the s domain:

$$
\begin{aligned}
& s X_{1}(s)-X_{1}(0) \\
= & \frac{X_{0} K_{0}}{s}\left(1-e^{-T s}\right)+K_{21} X_{2}(s)-\left(K_{12}+K_{e l}\right) X_{1}(s)
\end{aligned}
$$

$$
s X_{2}(s)-X_{2}(0)=K_{12} X_{1}(s)-K_{21} X_{2}(s)
$$

where: $X_{1}(0), X_{2}(0)$ are the initial conditions of $X_{1}(t), X_{2}(t)$.

$$
\text { At } t=0: \quad X_{1}(0)=X_{2}(0)=0
$$

Linear equation and solution are as follows:

$$
X_{1}(s)=\frac{X_{1}(0) s^{2}+\left(X_{0} K_{0}\left(1-e^{-T s}\right)+X_{1}(0) K_{21}\right) s+X_{0} K_{0} K_{21}\left(1-e^{-T s}\right)+X_{2}(0) K_{21}}{s\left(s^{2}+\left(K_{21}+K_{12}+K_{e l}\right) s+K_{21} K_{e l}\right)}=\frac{A_{1}}{s}-\frac{B_{1}}{s+\alpha}-\frac{C_{1}}{s+\beta}
$$

where:

$$
\begin{aligned}
& A_{1}=\frac{X_{0} K_{0} K_{21}}{\alpha \beta}\left(1-e^{-T s}\right), B_{1}=\frac{X_{0} K_{0}\left(K_{21}-\alpha\right)}{\alpha(\beta-\alpha)}\left(1-e^{-T s}\right), \\
& C_{1}=\frac{X_{0} K_{0}\left(K_{21}-\beta\right)}{\beta(\alpha-\beta)}\left(1-e^{-T s}\right), A_{1}=B_{1}+C_{1}
\end{aligned}
$$

and $\alpha, \beta$ are defined by:

$$
\begin{gathered}
s^{2}+\left(K_{21}+K_{12}+K_{e l}\right) s+K_{21} K_{e l}=(s+\alpha)(s+\beta), \text { then: } \\
\alpha=\frac{K_{12}+K_{21}+K_{e l}+\sqrt{\left(K_{12}+K_{21}+K_{e l}\right)^{2}-4 K_{21} K_{e l}}}{2} \\
\beta=\frac{K_{12}+K_{21}+K_{e l}-\sqrt{\left(K_{12}+K_{21}+K_{e l}\right)^{2}-4 K_{21} K_{e l}}}{2}
\end{gathered}
$$

Moreover,

$$
X_{2}(s)=\frac{X_{2}(0) s^{2}+\left(K_{12}+K_{e l}\right) X_{2}(0) s+K_{12} X_{1}(0) s+K_{12} X_{0} K_{0}\left(1-e^{-T s}\right)}{s\left(s^{2}+\left(K_{21}+K_{12}+K_{e l}\right) s+K_{21} K_{e l}\right)}=\frac{A_{2}}{s}-\frac{B_{2}}{s+\alpha}-\frac{C_{2}}{s+\beta}
$$

where:

$$
\begin{aligned}
X_{1}(t)= & B_{1}\left(\left(1-e^{-\alpha t}\right) u(t)-\left(1-e^{-\alpha(t-T)}\right) u(t-T)\right) \\
& +C_{1}\left(\left(1-e^{-\beta t}\right) u(t)-\left(1-e^{-\beta(t-T)}\right) u(t-T)\right) \\
X_{2}(t)= & B_{2}\left(\left(1-e^{-\alpha t}\right) u(t)-\left(1-e^{-\alpha(t-T)}\right) u(t-T)\right) \\
& +C_{2}\left(\left(1-e^{-\beta t}\right) u(t)-\left(1-e^{-\beta(t-T)}\right) u(t-T)\right)
\end{aligned}
$$$$
A_{2}=\frac{X_{0} K_{0} K_{21}}{\alpha \beta}\left(1-e^{-T s}\right), B_{2}=\frac{X_{0} K_{0} K_{21}}{\alpha(\beta-\alpha)}\left(1-e^{-T s}\right),
$$$$
C_{2}=\frac{X_{0} K_{0} K_{21}}{\beta(\alpha-\beta)}\left(1-e^{-T s}\right) \text { and } A_{2}=B_{2}+C_{2}
$$

Now, by the inverse Laplace transform, we have the solution for $X_{1}(t), X_{2}(t)$ in the time domain:

Then,

$$
\begin{gathered}
C_{1}=\frac{X_{1}}{V_{1}}=\frac{B_{1}\left(\left(1-e^{-\alpha t}\right) u(t)-\left(1-e^{-\alpha(t-T)}\right) u(t-T)\right)+C_{1}\left(\left(1-e^{-\beta t}\right) u(t)-\left(1-e^{-\beta(t-T)}\right) u(t-T)\right)}{V_{1}} \\
C_{2}=\frac{X_{2}}{V_{2}}=\frac{B_{2}\left(\left(1-e^{-\alpha t}\right) u(t)-\left(1-e^{-\alpha(t-T)}\right) u(t-T)\right)+C_{2}\left(\left(1-e^{-\beta t}\right) u(t)-\left(1-e^{-\beta(t-T)}\right) u(t-T)\right)}{V_{2}}
\end{gathered}
$$

\subsection{Derivations of Drug Concentration in Compartment after and Administration of IV Infusion Multiple Dose Using Laplace Transforms}

For a simulation of a 7 days treatment duration and 1 dose was administered a day, $u(t)-u(t-T)$ was replaced by $\sum_{k=0}^{6} u(t-24 k)-u(t-24 k-T)$ in the above differential equations (Equations (6) and (7)). And as a result, $\left(1-e^{-T S}\right)$ was replaced by $\sum_{k=0}^{6}\left(e^{-24 k s}-e^{-(24 k+T) s}\right)$ in its representation in the $s$ domain. Therefore, we had a similar solution for $X_{1}(t), X_{2}(t)$ as follows:

$$
\begin{aligned}
X_{1}(t)= & B_{1}\left(\sum_{k=0}^{6}\left(1-e^{-\alpha(t-24 k)}\right) u(t-24 k)\right. \\
& \left.-\left(1-e^{-\alpha(t-24 k-T)}\right) u(t-24 k-T)\right) \\
+ & C_{1}\left(\sum_{k=0}^{6}\left(1-e^{-\beta(t-24 k)}\right) u(t-24 k)\right. \\
& \left.-\left(1-e^{-\beta(t-24 k-T)}\right) u(t-24 k-T)\right)
\end{aligned}
$$




$$
\begin{aligned}
X_{2}(t)= & B_{2}\left(\sum_{k=0}^{6}\left(1-e^{-\alpha(t-24 k)}\right) u(t-24 k)\right. \\
& \left.-\left(1-e^{-\alpha(t-24 k-T)}\right) u(t-24 k-T)\right) \\
+ & C_{1}\left(\sum_{k=0}^{6}\left(1-e^{-\beta(t-24 k)}\right) u(t-24 k)\right. \\
& \left.-\left(1-e^{-\beta(t-24 k-T)}\right) u(t-24 k-T)\right)
\end{aligned}
$$

And, see Equations (8) and (9).

\subsection{Data Analysis}

Descriptive statistics of all levofloxacin PK parameters in healthy subjects and patients (mean, standard deviation, coefficient of variation) were obtained from literature. Pharmacokinetic simulation was then used to reveal the effect of input variables. A direct comparison of the experimental data (mean $\pm \mathrm{sd}$ ) obtained from simulation was involved. Comparison between the experimental and observed data from various studies reported in literature was conducted using a student t-test. Mean and standard deviations of PK parameters were determined to assess how well the model described the clinical data. The best PK model and log-normal distribution (mean and variance) of all the transfer rates were then used as input in the Monte Carlo simulation process to generate plasma and tissue concentrations.

\subsection{Matlab/Simulink Monte Carlo Simulations}

A 2-compartment open model without a lag time was used to generate each concentration-time profile, where drug distribution rates $\left(K_{12}, K_{21}\right)$ or drug elimination rate $\left(K_{e l}\right)$ were random variables associated with their distribution information. Their distributions were considered lognormal distributions. The mean concentration-time profile was generated by using mean values of PK parameters.

\subsection{Study the Effect of Elimination and Distribution Rate on Drug Level in Plasma and Tissue}

Investigation of varying the effect of elimination and distribution rates was performed using various values of those parameters. Monte Carlo simulation of 5000 drug concentration profiles was performed by importing elimination/distribution rates randomly into pharmacokinetic model and according gain blocks as in Figure 1. Random values were chosen from their associated log-normal distributions (mean and standard deviation) of reference values.

\subsection{Simulink for Single and Multiple Dose IV Infusion Administration}

Random function (rand) was used to randomly select pharmacokinetic parameter values which were associated to their distribution. The bounds for each PK parameter were set in accordance with the pharmacokinetic parameter values obtained. Simulink (Matlab, Natick, Massachusetts, USA) was used to simulate signals and determine how these concentrations vary over time using a system of 2 differential equations to describe plasma concentrations in compartment 1 and tendon concentration were in compartment 2.

The block Plasma $\left(X_{1}\right)$ and Tissue $\left(X_{2}\right)$ were two integrators. They took the integration of the inputs $\mathrm{dX} 1 / \mathrm{dt}$, $\mathrm{dX} 2 / \mathrm{dt}$ and returned the outputs $X_{1}$ and $X_{2}$. The block Pulse Generator 1 was to reset the integrator plasma compartment each 24 time steps (corresponding to 24 hours per day). The block Pulse Generator was to describe multiple doses: One dose oral or infusion calculated as amount over the time of infusion or duration of absorption (1 time step) dose per 24 hours (24 time steps). Other blocks including gain blocks $\left(K_{12}, K_{21}, K_{e l}, 1 / V_{1}\right.$, $1 / V_{2}$ ) and sum blocks were to implement the left hand side of each of two differential equations. Concentration time profiles in two compartments were obtained in two scope blocks by running this simulation.

\section{Results}

\subsection{Simulated Levofloxacin Concentration Time Profiles Following a $1 \mathrm{~h}$-Infusion and Oral $500 \mathrm{mg}$ Single Dose Administration}

Plasma and well-perfused organs such as lung, skin, and

$$
\begin{gathered}
C_{1}=\frac{X_{1}}{V_{1}}=\left[\begin{array}{l}
B_{1}\left(\sum_{k=0}^{6}\left(1-e^{-\alpha(t-24 k)}\right) u(t-24 k)-\left(1-e^{-\alpha(t-24 k-T)}\right) u(t-24 k-T)\right) \\
+C_{1}\left(\sum_{k=0}^{6}\left(1-e^{-\beta(t-24 k)}\right) u(t-24 k)-\left(1-e^{-\beta(t-24 k-T)}\right) u(t-24 k-T)\right)
\end{array}\right] / V_{1} \\
C_{2}=\frac{X_{2}}{V_{2}}=\left[\begin{array}{l}
B_{2}\left(\sum_{k=0}^{6}\left(1-e^{-\alpha(t-24 k)}\right) u(t-24 k)-\left(1-e^{-\alpha(t-24 k-T)}\right) u(t-24 k-T)\right) \\
+C_{2}\left(\sum_{k=0}^{6}\left(1-e^{-\beta(t-24 k)}\right) u(t-24 k)-\left(1-e^{-\beta(t-24 k-T)}\right) u(t-24 k-T)\right)
\end{array}\right] / V_{2}
\end{gathered}
$$




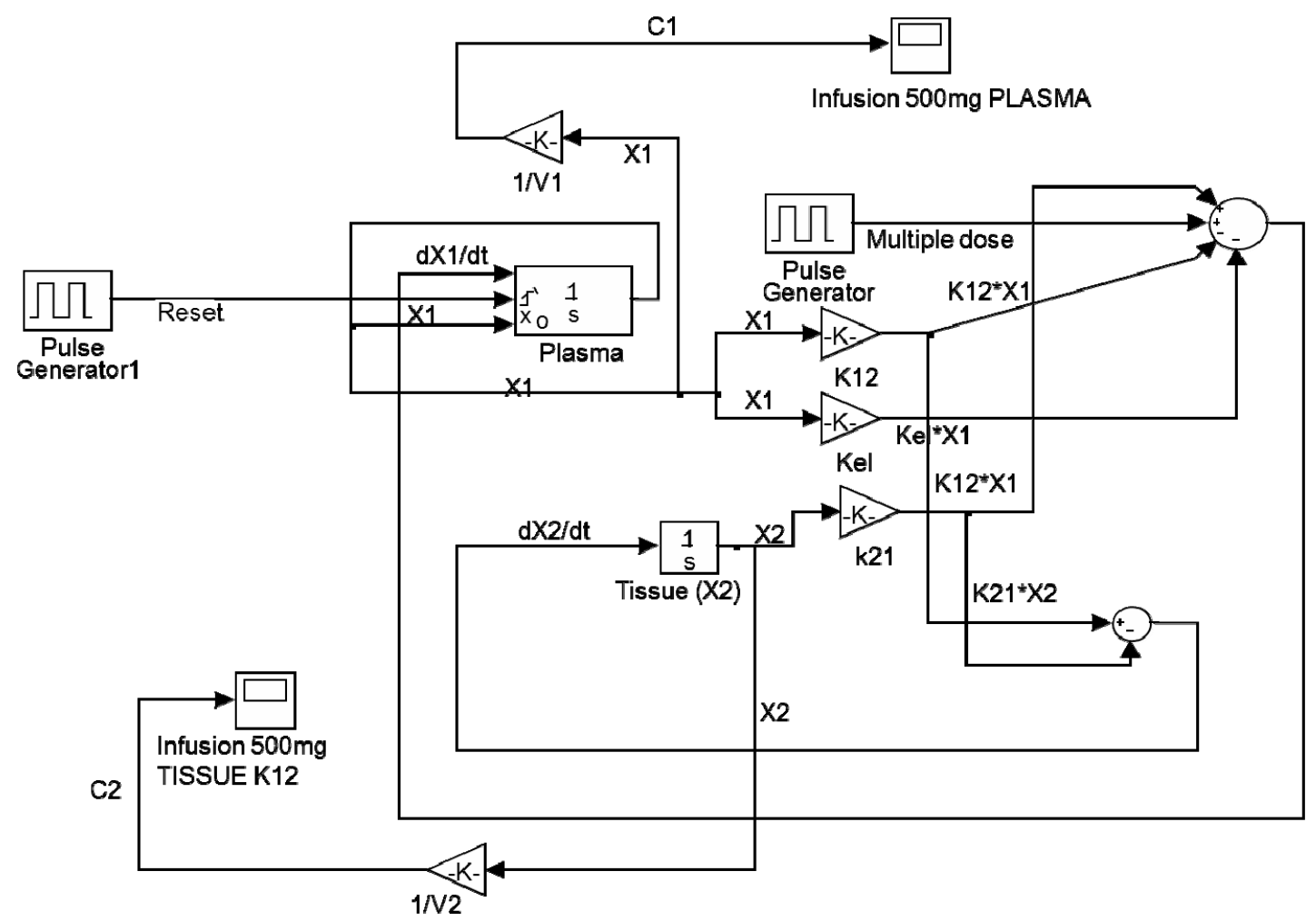

Figure 1. Simulink for intravenous infusion 1 hour q24 for 7 days.

spongiosa etc. were grouped in compartment 1 . Tissues characterized by poor blood flow such as tendon tissue (including Achilles tendon) along with adipose and cartilage were grouped in compartment 2. Tendon was anticipated to have very low redistribution rate compared to other sites, and was grouped in compartment 2 where the distribution rate is comparable to those of cortical bone and adipose tissue $[9,11,13]$.

Figure 2 shows that generated drug concentration time curves were approximately super-imposable in plasma and in tissue after oral administration and IV infusion. This is in agreement with clinical data when approximately $100 \%$ drug absorption occurs in the oral route. The drug peak concentration produced with IV infusion was higher than that of oral administration. This initial simulation of the average drug concentration ratio between plasma and tissue showed no obvious change with time $4 \mathrm{hr}$ post dosing onward, and that equilibrium between plasma and tissue drug concentrations were actually achieved $4 \mathrm{hr}$ after dosing and the tissue concentrations declined proportionally to the plasma concentrations.

In addition, simulated data also show that after a single dose, drug concentration values in tissue achieved at Cmax of $3.08 \mu \mathrm{g} / \mathrm{mL}$ and $3.28 \mu \mathrm{g} / \mathrm{mL}$, and $\mathrm{Tmax}$ of 3.25 hrs and $3.11 \mathrm{hr}$, and AUC0-24 of $42.05 \mu \mathrm{g} \cdot \mathrm{hr} / \mathrm{mL}$ and $42.91 \mu \mathrm{g} \cdot \mathrm{hr} / \mathrm{mL}$ for $500 \mathrm{mg}$ oral dose and IV infusion, respectively.

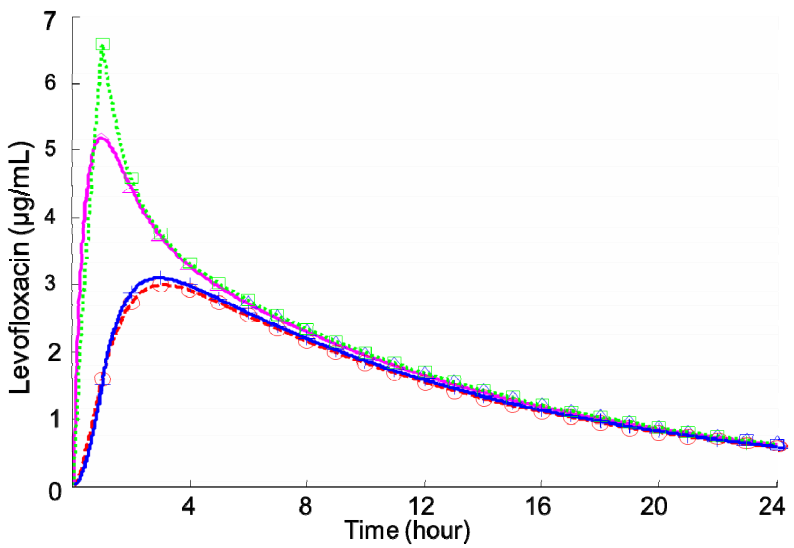

Figure 2. Simulated mean plasma and tissue levofloxacin concentration-time profiles following the oral and IV infusion administrations of a single $500 \mathrm{mg}$ dose. Key [... $\square]$ : drug concentrations in plasma; $[\ldots+]$ : drug concentrations in tissue after a single intravenous infusion; $[-\Delta]$ : drug concentrations in plasma, $[-0]$ : drug concentrations in tissue after a single oral dosing.

\subsection{Simulated Levofloxacin Drug Concentration Time Profiles after Multiple $500 \mathrm{mg}$ Once Daily Dosing Administration}

The simulated data show that plasma and tissue Levofloxacin concentrations after repeated doses for 7 days resemble the drug concentration profile of a single dose well. Only a slight accumulation of the drug is observed 
on the second dose onward. The concentration time curves after each dose for a 7-days treatment course are presented in Figure 3(a) (oral route) and Figure 3(b) (intravenous infusion). At steady state, simulated mean peak and trough plasma concentration values $\left(\mathrm{C}_{\max }, \mathrm{C}_{\min }\right.$, respectively) following once-daily administration regimen are in agreement with reported values (Table 2). In addition, simulated data for compartment 1 represent plasma concentrations after consecutive doses confirms Levofloxacin accumulates minimally following repeated once-daily administration dosing regimen. Simulated data also show that after multiple doses, the mean Cmax in tissue achieved concentrations of $4.08 \mu \mathrm{g} / \mathrm{mL}$ and 5.12 $\mu \mathrm{g} / \mathrm{mL}$ with Tmax of $3.5 \mathrm{hr}$ and $3.14 \mathrm{hr}$, and AUC0-24 of $42.05 \mu \mathrm{g} \cdot \mathrm{hr} / \mathrm{mL}$ and $42.91 \mu \mathrm{g} \cdot \mathrm{hr} / \mathrm{mL}$ for multiple oral and IV doses, respectively.

\subsection{Comparison of Pharmacokinetic Values between the Simulated and Observed Data}

Comparison of pharmacokinetic values in plasma (compartment 1) between the simulated data and observed data after a single dose of $500 \mathrm{mg}$ Levofloxacin as well as after multiple-dose 1-hour intravenous infusion and oral administration of levofloxacin is presented in Table 2. The results show acceptable agreement with published data. The chosen pharmacokinetic model that was used to simulate the drug concentration time curves was an excellent fit to the observed plasma concentration data.

The predicted concentrations of Levofloxacin obtained in soft tissue (compartment 2) after an administration of a single $500 \mathrm{mg}$ of the compound at $3.0-3.1 \mathrm{hr}$ are around $3.2 \mu \mathrm{g} / \mathrm{mL}$ for IV administration and $3.08 \mu \mathrm{g} / \mathrm{mL}$ for oral route. The simulated results in the tissue (compartment 2) are in good agreement to Levofloxacin concentration in clinical settings (in adipose tissue: $3.1 \mu \mathrm{g} / \mathrm{mL}$ at $180 \mathrm{~min}$ after administration) $[19,20]$. Simulated drug concentrations in this study also produce a mean $\mathrm{C}_{\max }$ in the tissue (compartment 2) approximately $41 \%$ that of plasma for IV administration and 50.6\% for oral route. These data are in agreement with observed clinical data as Levofloxacin also possesses the capacity to penetrate to cortical bone (an organ included in the tissue or compartment 2) of $36 \%-100 \%$ of the plasma concentration after a single intravenous dose of $500 \mathrm{mg}$ [9,20-22].

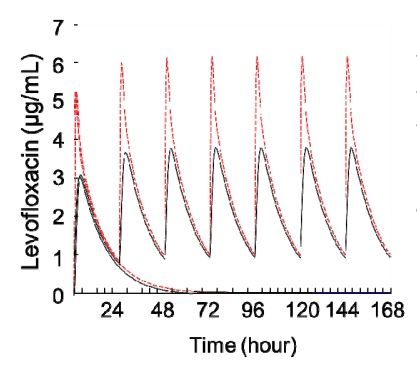

(a) oral route

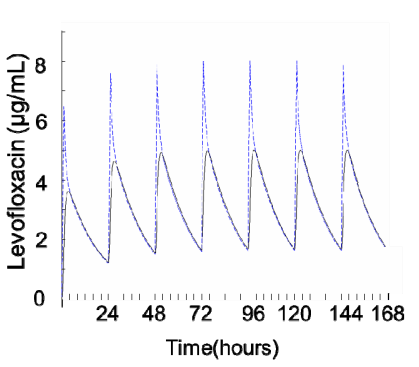

(b) intravenous infusion
Figure 3. Simulated Levofloxacin Concentration Time Profiles following multiple doses. (a) Graphs show the concentration curves of once daily $500 \mathrm{mg}$ oral administration for plasma (dashed Red) and tissue (Black); (b) Graphs display the concentration curves of once daily $500 \mathrm{mg} 1 \mathrm{~h}$ infusion administration for plasma (dashed Blue) and tissue (Black).

Table 2. Mean pharmacokinetic parameters of simulated and observed Levofloxacin concentrations. (A), the compartment 1 concentration after a single $500 \mathrm{mg}$ dose, and (B), plasma concentration after consecutive 7 doses of once daily administrations. Similarity is observed between simulation and clinical data. Clinical data are mean $\pm S D$. $C_{\max }=$ maximum concentration. $T_{\max }=$ time to achieve $C_{\max }$. AUC0-24 = area under the concentration-time curve from 0 - 24 hours.

\begin{tabular}{|c|c|c|c|c|}
\hline \multicolumn{5}{|c|}{ (A) Single dose } \\
\hline \multirow[b]{2}{*}{ Plasma } & \multicolumn{2}{|c|}{$500 \mathrm{mg}$ intravenous infusion } & \multicolumn{2}{|c|}{$500 \mathrm{mg}$ oral } \\
\hline & Simulated data & Clinical data [23] & Simulated data & Clinical data [23] \\
\hline $\mathrm{C}_{\max }(\mu \mathrm{g} / \mathrm{mL})$ & 6.5956 & $6.4 \pm 0.8$ & 5.19 & $5.2 \pm 1.4$ \\
\hline $\mathrm{T}_{\max }$ & 1 & 1 & 1 & $1 \pm 0.4$ \\
\hline AUC0-24 ( $\mu \mathrm{g} \cdot \mathrm{hr} / \mathrm{mL})$ & 53.77 & $54.6 \pm 11.1$ & 48.82 & $47.5 \pm 6.7$ \\
\hline Half-life (hr) & 7.0 & $7.0 \pm 0.8(\mathrm{Kel}=0.099)$ & 7.6 & $7.6 \pm 1.6(\mathrm{Kel}=0.091)$ \\
\hline \multicolumn{5}{|c|}{ (B) Multiple doses } \\
\hline & \multicolumn{2}{|c|}{$500 \mathrm{mg}$ intravenous infusion 7 days } & \multicolumn{2}{|c|}{$500 \mathrm{mg}$ oral 7 days } \\
\hline Plasma & Simulated data & Clinical data [24] & Simulated data & Clinical data [24] \\
\hline $\mathrm{C}_{\max }(\mu \mathrm{g} / \mathrm{mL})$ & 8.023 & 7.9 & 6.08 & $5.7 \pm 1.4$ \\
\hline $\mathrm{T}_{\max }$ & 1 & 1 & 1.1 & 1.1 \\
\hline Half-life & 6.8 & 6.8 & 6.8 & 6.8 \\
\hline $\mathrm{Cmin}(\mu \mathrm{g} / \mathrm{mL})$ & & & & $0.5 \pm 0.2$ \\
\hline
\end{tabular}




\subsection{The Effect of Elimination Rate on Levofloxacin Concentration Profiles}

Five thousand possible values of the elimination rate constant were randomly selected from its log normal distribution to generate associated individual plasma and tissue drug concentration profiles (Figure 4(a)). In the patients with impaired renal function, who are expected to have low rates of elimination, the drug concentrations in plasma could rise as high as $35 \mu \mathrm{g} / \mathrm{mL}$ at day 7 (compared to a concentration of $6-7 \mu \mathrm{g} / \mathrm{mL}$ after 7 days of patients with normal renal function, shown in Figure 3). Drug concentrations in tissue were affected by renal function, but did not rise as high as in the plasma. After 7 doses, drug concentration in tissue rose to $12 \mu \mathrm{g} / \mathrm{mL}$. Therefore, the elimination process has less effect on raising drug concentrations in the tissue compared to that in the plasma compartment. Furthermore, because of dosing adjustment when Levofloxacin is given to patients with renal impairment, drug concentrations in tissue would not be expected to accumulate as high as $12 \mu \mathrm{g} / \mathrm{mL}$.

\subsection{Effect of Varying the Distribution Rate Constant on Plasma to Tissue Drug Concentrations}

Assessing the variation of drug concentration at tendon site was done by varying the values of redistribution rates while other PK parameters were keep constant at mean values. 5000 values of the rate constant of drug distribution from compartment 1 to compartment $2\left(K_{12}\right)$ were randomly generated from its log-normal distribution (mean, standard deviation) with the assumption that pa- tients had normal renal function. Figure 4(b) shows the results of simulated concentration time drug profiles in plasma and in tissue after 7 doses of Levofloxacin. The accumulation of the drug in tissue significantly increases after the second dose. After the third dose, the concentration in tissue is higher than in plasma and can be up to $9.5 \mu \mathrm{g} / \mathrm{mL}$. Compared to the highest tissue drug concentration (shown in Figure 3(b)) for normal subjects after 7 doses is only $4.5 \mu \mathrm{g} / \mathrm{mL}$ suggesting a difference of 5.0 $\mu \mathrm{g} / \mathrm{mL}$ between the two groups or more than 2 times higher drug concentration in tissue when distribution changes, and the concentration in tissue is even higher than in plasma.

Tendon was anticipated to have very low redistribution rate $\left(K_{21}\right)$ compared to other organs which were also grouped in compartment 2 , while its distribution rate be comparable to tissues of compartment 2 . The concentrations in tendon would fall in the higher range of tissue concentrations. Drug concentrations in tissue could increase dramatically from an average of $5 \mu \mathrm{g} / \mathrm{mL}$ up to 9.5 $\mu \mathrm{g} / \mathrm{mL}$ when factors such as increased tissue binding or blood flow occur. The high values of Levofloxacin in tissue could be drug concentrations in tendon and be the reason for drug toxicity to the tendon.

\section{Discussion}

Levofloxacin-associated tendinopathy, similar to other Fluoroquinolones, is a widely recognized event that eventually led the US Food and Drug Administration to add a black box warning label citing the risk of tendonitis and tendon rupture [15]. Pharmacokinetic studies have

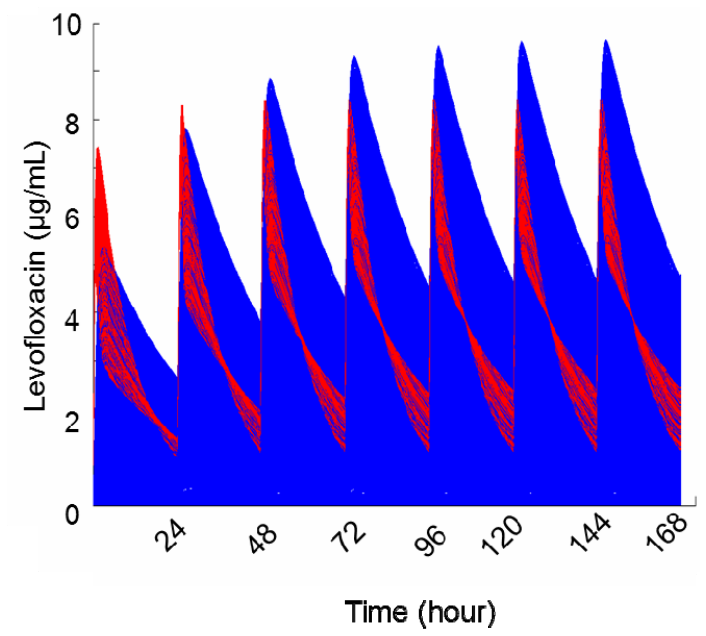

(b)

Figure 4. (a) Effect of decreased elimination rate constants on the drug concentration in plasma and tissue after oral administration of Levofloxacin. Reduction in elimination rate can bring the accumulation of concentration in plasma (Red) to 35 $\mu \mathrm{g} / \mathrm{mL}$ at day 7 while does not add much the compound into tissue (Blue). (b) Simulated drug concentration time profiles when $K_{12}$ is varied after oral administration of 7 doses with $K_{12}$ being varied. Five thousand drug profiles in plasma (Red) and in tissue (Blue) present a scenario in which accumulation of drug in tissue significantly increases after the second dose and is higher than plasma after the third dose of the drug. 
been studied to address issues such as drug interaction, systemic availability and disposition of Levofloxacin in healthy adults, the elderly and patients with renal impairment [25]. The plasma concentration data from intravenous administration of Levofloxacin from a multicenter open-label study in patients with bacterial infection showed that a two compartment open model (firstorder elimination from the central compartment and zeroorder intravenous infusion and absorption duration) was the best fit. When the absorption process is rapid, pharmacokinetic modeling is more sampling schedule subjective and there may not be enough sampling time points to allow definite assessment the absorptive phase. Therefore, in this study, a rapid first order oral absorption rate was adopted because it was determined to be the best model to simulate drug concentration time profiles in plasma. Pharmacokinetic values were similar to values in subjects without bacterial infections (healthy subjects) in phase I clinical studies. Creatinine clearance, age and race were included in the demographic model for prediction of Levofloxacin clearance in the subjects, with creatinine clearance explaining most of the population variance. The elimination of Levofloxacin is mainly affected by the degree of renal function. Thus, dosage adjustment is required in subjects with renal impairment [26].

This study is the first to suggest that the potential concentration accumulation in tendon tissue may be extremely and unexpectedly high $(9.5 \mu \mathrm{g} / \mathrm{mL})$ in some patients as compared to measured plasma concentrations. The concentration gradient transport and high vascular blood flow are the most likely mechanisms for rapid accumulation of Levofloxacin in compartment-1 categorized organs (liver, kidney and lung). The predicted concentrations in such organs also correspond to the actual concentrations seen in reported studies where the drug concentrations in those areas achieved peak concentration in a short time similar to that seen in plasma, and was eliminated more quickly versus the tissues in compartment-2 which experienced longer exposure $[10,11]$. A scenario is also seen in Figure 4(b) where the accumulation of drug in tendon tissue happened and rises higher than the plasma concentrations after 7 days of treatment. Clinically, it is believed that greater drug diffusion into tissue incurs higher intrinsic tenotoxicity in the case of Ofloxacin and Levofloxacin, where the two compounds show similar intrinsic tenotoxicity and diffusion of drug into tissue [27]. It is also reported that intra-articular Ofloxacin concentration in juvenile dogs was approximately 1.8 to 2.0 times higher than serum concentrations $2 \mathrm{hr}$ after dosing [28]. Because Levofloxacin shares characteristics with Ofloxacin in terms of intracellular concentration, it is more toxic than Ciprofloxacin due to higher diffusion of drug into tissue $[27,29$, 30].
Another mechanism that may also contribute to the toxicity of Levofloxacin to the Achilles tendon is the ratio of influx and efflux to the tendon cells from the extracellular fluid. The reported existence of very poor efflux out of the cells through plasma membrane [31] leads to the possibility that large doses of Levofloxacin that typically produce concentrations of 6 to $7 \mathrm{mg} / \mathrm{L}$ or more in which efflux would largely be impaired resulting in greater-than-anticipated drug accumulation in tendon producing greater potential toxicities associated with higher cellular and tissue accumulation of the compound [31]. One can connect this suggestion to the scenario seen in clinics where the majority of adverse tendon incidents is observed around 8 days of treatment with consecutive doses, although symptoms can occur as early as two hours after the first dose $[14,32,33]$. This is consistent with data on file that the concentration increases significantly with higher or repeated doses $[34,35]$.

Accumulation of Fluoroquinolones have been reported in circulating and local blood leukocytes [36-40] where the uptake of Levofloxacin in cells was dependent on peripheral concentrations and higher concentrations of about 4 to 6 times was observed in intracellular tissues compared to extracellular fluid $[39,41]$. Interestingly, low $\mathrm{Mg}^{2+}$ and $\mathrm{Ca}^{2+}$ concentrations were able to enhance the cellular concentration and toxicity of the compound to both blood and tendon cells $[42,43]$. The potential mechanism for adverse events due to accumulation of Levofloxacin in tendon cells was similar to that observed in leukocytes. In the case of Achilles tendon, disorders were also characterized by edema that possibly led to the observed increased permeability in blood vessels, increased inflammation and eukaryotic chemotaxis [44]. This produces chemo-attractants resulting in the accumulation of phagocytic blood cells which may further convey Levofloxacin into the lesions leading to additional compound appearing in the lesions, together with phagocytic actions, which produces further harm to tissue leading to orthopedic incidents. Moreover, Levofloxacin also causes significant cell death, activation of extracellular matrix metalloproteinase activities and marked reduction of collagen content in tendon which significantly weakens the tendon structure even with lower doses compared to other members of the drug family $[27,45]$.

Together with above reasons, physiological and anatomic structure of Achilles tendon can also be contributors to help explain the reason why rupture tends to happen at Achilles tendon but very rarely in other tendons [46]. It is worth noting that in addition to the poor blood supply in the center of Achilles tendon, which can be exacerbated with Levofloxacin, the tendons also have to support body weight and are responsible for walking and running, which may increase stress to tendons by up to 12.5 times body weight $[13,47]$. The results presented are 
in agreement with reported cases of Achilles tendonitis and ruptures, where most of the symptoms were reported when patients were walking or involved in sporting activities. Halting the use of Levofloxacin together with resting alone or resting in combination with supportive treatments can relieve, prevent or reduce the severity of adverse events $[7,15,46,48]$. In fact, histological studies have observed that the pathological features found in tendon disorders produced by Fluoroquinolones are similar to those seen in excessive exercise injuries in athletes, suggesting that rapid and immense stretching activities may in part play a significant role in the incidents [46, 49].

\section{Conclusion}

The rationale of this research is to use simulation to demonstrate that the incidents can be predicted based upon appropriate given information with reliable accuracy leading to the appropriate use of the drug and thereby prevent complications. In fact, there is a suspected population where Levofloxacin penetration is characteristically much higher than the typical population suggesting increased drug distribution into tendon produces interference of genetic platforms. Nevertheless, the findings of this simulation model need to be further confirmed by experimental studies in the light of plasma and local concentrations personalized to individual characteristics. The limitations of the studies include lack of in vitro and in vivo information on distribution of Levofloxacin to support the observations in this model. However, the model is readily adaptable to include influx and efflux parameters. A similar application has proven useful in prediction of the concentrations of a Fluoroquinolone in bone tissue of patients undergoing hip replacement [50] suggesting the usefulness and practicality of pharmacokinetic simulation.

\section{REFERENCES}

[1] A. Durey, et al., "Levofloxacin-Induced Achilles Tendinitis in a Young Adult in the Absence of Predisposing Conditions," Yonsei Medical Journal, Vol. 51, No. 3, 2010, pp. 454-456. doi:10.3349/ymj.2010.51.3.454

[2] J. B. Kahn, "Latest Industry Information on the Safety Profile of Levofloxacin in the US," Chemotherapy, Vol. 47, Suppl. 3, 2001, pp. 32-37. doi:10.1159/000057842

[3] A. S. Mathis, et al., "Levofloxacin-Associated Achilles Tendon Rupture," Annals of Pharmacotherapy, Vol. 37, No. 7-8, 2003, pp. 1014-1017. doi:10.1345/aph.1C505

[4] A. Melhus, et al., "Levofloxacin-Associated Achilles Tendon Rupture and Tendinopathy," Scandinavian Journal of Infectious Diseases, Vol. 35, No. 10, 2003, pp. 768-770. doi:10.1080/00365540310015863

[5] J. R. Lewis, J. G. Gums and D. L. Dickensheets,
"Levofloxacin-Induced Bilateral Achilles Tendonitis," Annals of Pharmacotherapy, Vol. 33, No. 7-8, 1999, pp. 792-795. doi:10.1345/aph.18298

[6] F. Fleisch, K. Hartmann and M. Kuhn, "Fluoroquinolone-Induced Tendinopathy: Also Occurring with Levofloxacin," Infection, Vol. 28, No. 4, 2000, pp. 256-257. doi: $10.1007 / \mathrm{s} 150100070050$

[7] L. J. Haddow, et al., "Spontaneous Achilles Tendon Rupture in Patients Treated with Levofloxacin," Journal of Antimicrobial Chemotherapy, Vol. 51, No. 3, 2003, pp. 747-748. doi:10.1093/jac/dkg081

[8] C. Parmar and K. P. Meda, "Achilles Tendon Rupture Associated with Combination Therapy of Levofloxacin and Steroid in Four Patients and a Review of the Literature," Foot \& Ankle International, Vol. 28, No. 12, 2007, pp. 1287-1289. doi:10.3113/FAI.2007.1287

[9] H. von Baum, et al., "Tissue and Serum Concentrations of Levofloxacin in Orthopaedic Patients," International Journal of Antimicrobial Agents, Vol. 18, No. 4, 2001, pp. 335-340. doi:10.1016/S0924-8579(01)00423-X

[10] M. A. Zeitlinger, et al., "A Pilot Study Testing Whether Concentrations of Levofloxacin in Interstitial Space Fluid of Soft Tissues May Serve as a Surrogate for Predicting Its Pharmacokinetics in Lung," International Journal of Antimicrobial Agents, Vol. 29, No. 1, 2007, pp. 44-50. doi:10.1016/j.ijantimicag.2006.08.045

[11] K. De Angelis, et al., "Blood Flow Measurements in Rats Using Four Color Microspheres during Blockade of Different Vasopressor Systems," Brazilian Journal of Medical and Biological Research, Vol. 38, No. 1, 2005, pp. 119-125. doi:10.1590/S0100-879X2005000100018

[12] D. M. Boothe, et al., "Tissue Concentrations of Enrofloxacin and Ciprofloxacin in Anesthetized Dogs Following Single Intravenous Administration," Veterinary Therapeutics, Vol. 2, No. 2, 2001, pp. 120-128.

[13] M. N. Doral, et al., "Functional Anatomy of the Achilles Tendon," Knee Surgery, Sports Traumatology, Arthroscopy, Vol. 18, No. 5, 2010, pp. 638-643. doi:10.1007/s00167-010-1083-7

[14] J. M. Casparian, et al., "Quinolones and Tendon Ruptures," Southern Medical Journal, Vol. 93, No. 5, 2000, pp. 488-491.

[15] M. M. Hall, J. T. Finnoff and J. Smith, "Musculoskeletal Complications of Fluoroquinolones: Guidelines and Precautions for Usage in the Athletic Population," $P M \& R$, Vol. 3, No. 2, 2011, pp. 132-142. doi:10.1016/j.pmri.2010.10.003

[16] Y. Kashida and M. Kato, "Characterization of Fluoroquinolone-Induced Achilles Tendon Toxicity in Rats: Comparison of Toxicities of 10 Fluoroquinolones and Effects of Anti-Inflammatory Compounds," Antimicrobial Agents and Chemotherapy, Vol. 41, No. 11, 1997, pp. 2389-2393.

[17] P. D. van der Linden, et al., "Increased Risk of Achilles Tendon Rupture with Quinolone Antibacterial Use, Especially in Elderly Patients Taking Oral Corticosteroids," Archives of Internal Medicine, Vol. 163, No. 15, 2003, pp. 1801-1807. doi:10.1001/archinte.163.15.1801 
[18] Levaquin NDA 020634 Approval Package Study \#K09077 and LOFBIV-MULT-001, 2012. http://www.accessdata.fda.gov/drugsatfda docs/nda/96/0 20634_levaquin_toc.cfm

[19] R. Bellmann, et al., "Tissue Pharmacokinetics of Levofloxacin in Human Soft Tissue Infections," British Journal of Clinical Pharmacology, Vol. 57, No. 5, 2004, pp. 563-568. doi:10.1111/j.1365-2125.2004.02059.x

[20] T. Rimmele, et al., "Diffusion of Levofloxacin into Bone and Synovial Tissues," Journal of Antimicrobial Chemotherapy, Vol. 53, No. 3, 2004, pp. 533-535. doi: $10.1093 / \mathrm{jac} / \mathrm{dkh} 110$

[21] C. B. Landersdorfer, et al., "Penetration of Antibacterials Into Bone: Pharmacokinetic, Pharmacodynamic and Bioanalytical Considerations," Clinical Pharmacokinetics, Vol. 48, No. 2, 2009, pp. 89-124. doi:10.2165/00003088-200948020-00002

[22] F. Pea, "Penetration of Antibacterials Into Bone: What Do We Really Need to Know for Optimal Prophylaxis and Treatment of Bone and Joint Infections?" Clinical Pharmacokinetics, Vol. 48, No. 2, 2009, pp. 125-127. doi:10.2165/00003088-200948020-00003

[23] S. C. Chien, et al., "Pharmacokinetic Profile of Levofloxacin Following Once-Daily 500-Milligram Oral or Intravenous Doses," Antimicrobial Agents and Chemotherapy, Vol. 41, No. 10, 1997, pp. 2256-2260.

[24] J. E. Mazuski, et al., "The Surgical Infection Society Guidelines on Antimicrobial Therapy for Intra-Abdominal Infections: Evidence for the Recommendations," Surgical Infections, Vol. 3, No. 3, 2002, pp. 175-233. doi:10.1089/109629602761624180

[25] New Drug Application 20-634, 2012. http://www.accessdata.fda.gov/scripts/cder/drugsatfda/ind ex.cfm?fuseaction=Search.Label_ApprovalHistory\#apphi st

[26] Levaquin NDA 020634 Label, 2012.

[27] F. Pouzaud, et al., "In Vitro Discrimination of Fluoroquinolones Toxicity on Tendon Cells: Involvement of Oxidative Stress," Journal of Pharmacology and Experimental Therapeutics, Vol. 308, No. 1, 2004, pp. 94-402.

[28] K. Yabe, et al., "A Non-Arthropathic Dose and Its Disposition Following Repeated Oral Administration of Ofloxacin, a New Quinolone Antimicrobial Agent, to Juvenile Dogs," Journal of Veterinary Medical Science, Vol. 63, No. 8, 2001, pp. 867-872. doi:10.1292/jvms.63.867

[29] A. Meissner, K. Borner and P. Koeppe, "Concentrations of Ofloxacin in Human Bone and in Cartilage," Journal of Antimicrobial Chemotherapy, Vol. 26, Suppl. D, 1990, pp. 69-74.

[30] P. D. van der Linden, et al., "Tendon Disorders Attributed to Fluoroquinolones: A Study on 42 Spontaneous Reports in the Period 1988 to 1998," Arthritis \& Rheumatism, Vol. 45, No. 3, 2001, pp. 235-239. doi:10.1002/1529-0131(200106)45:3<235::AID-ART254 $\geq 3.0 . \mathrm{CO} ; 2-7$

[31] J. M. Michot, et al., "Influence of Efflux Transporters on the Accumulation and Efflux of Four Quinolones (Ciprofloxacin, Levofloxacin, Garenoxacin, and Moxifloxacin) in J774 Macrophages," Antimicrobial Agents and Chemotherapy, Vol. 49, No. 6, 2005, pp. 2429-2437. doi:10.1128/AAC.49.6.2429-2437.2005

[32] J. T. Jagose, et al., "Achilles Tendon Rupture Due to Ciprofloxacin," New Zealand Medical Journal, Vol. 109, No. 1035, 1996, pp. 471-472.

[33] Y. Khaliq and G. G. Zhanel, "Fluoroquinolone-Associated Tendinopathy: A Critical Review of the Literature," Clinical Infectious Diseases, Vol. 36, No. 11, 2003, pp. 1404-1410. doi:10.1086/375078

[34] M. H. Gotfried, L. H. Danziger and K. A. Rodvold, "Steady-State Plasma and Intrapulmonary Concentrations of Levofloxacin and Ciprofloxacin in Healthy Adult Subjects," Chest, Vol. 119, No. 4, 2001, pp. 1114-1122. doi:10.1378/chest.119.4.1114

[35] S. Swoboda, et al., "Tissue and Serum Concentrations of Levofloxacin $500 \mathrm{mg}$ Administered Intravenously or Orally for Antibiotic Prophylaxis in Biliary Surgery," Journal of Antimicrobial Chemotherapy, Vol. 51, No. 2, 2003, pp. 459-462. doi:10.1093/jac/dgk056

[36] A. Boeckh, et al., "Time Course of Enrofloxacin and Its Active Metabolite in Peripheral Leukocytes of Dogs," Veterinary Therapeutics, Vol. 2, No. 4, 2001, pp. 334344.

[37] M. B. Carlier, et al., "Cellular Uptake, Localization and Activity of Fluoroquinolones in Uninfected and Infected Macrophages," Journal of Antimicrobial Chemotherapy, Vol. 26, Suppl. B, 1990, pp. 7-39.

[38] P. Schuler, et al., "Penetration of Sparfloxacin and Ciprofloxacin into Alveolar Macrophages, Epithelial Lining Fluid, and Polymorphonuclear Leucocytes," European Respiratory Journal, Vol. 10, No. 5, 1997, pp. 1130-1136. doi:10.1183/09031936.97.10051130

[39] K. Taira, H. Koga and S. Kohno, "Accumulation of a Newly Developed Fluoroquinolone, OPC-17116, by Human Polymorphonuclear Leukocytes," Antimicrobial Agents and Chemotherapy, Vol. 37, No. 9, 1993, pp. 18771881. doi:10.1128/AAC.37.9.1877

[40] P. M. Tulkens, "Accumulation and Subcellular Distribution of Antibiotics in Macrophages in Relation to Activity against Intracellular Bacteria," Ciprofloxacinn in Pulmonology, San Francisco, 1990.

[41] R. P. Smith, et al., "Levofloxacin Penetrates Human Monocytes and Enhances Intracellular Killing of Staphylococcus aureus and Pseudomonas aeruginosa," Journal of Antimicrobial Chemotherapy, Vol. 45, No. 4, 2000, pp. 483-488. doi:10.1093/jac/45.4.483

[42] M. Egerbacher, B. Wolfesberger and C. Gabler, "In Vitro Evidence for Effects of Magnesium Supplementation on Quinolone-Treated Horse and Dog Chondrocytes," Veterinary Pathology, Vol. 38, No. 2, 2001, pp. 143-148. doi:10.1354/vp.38-2-143

[43] D. Vazifeh, A. Bryskier and M. T. Labro, "Mechanism underlying levofloxacin Uptake by human polymorphonuclear neutrophils," Antimicrobial Agents and Chemotherapy, Vol. 43, No. 2, 1999, pp. 246-252.

[44] M. Kato, et al., "Histological Examination on Achilles Tendon Lesions Induced by Quinolone Antibacterial 
Agents in Juvenile Rats," Toxicologic Pathology, Vol. 23, No. 3, 1995, pp. 85-392.

doi: $10.1177 / 019262339502300315$

[45] R. J. Williams III, et al., "The Effect of Ciprofloxacin on Tendon, Paratenon, and Capsular Fibroblast Metabolism," American Journal of Sports Medicine, Vol. 28, No. 3, 2000, pp. 364-369.

[46] G. K. Kim, "The Risk of Fluoroquinolone-Induced Tendinopathy and Tendon Rupture: What Does the Clinician Need to Know?" Journal of Clinical and Aesthetic Dermatology, Vol. 3, No. 4, 2010, pp. 49-54.

[47] P. Szaro, et al., "Fascicles of the Adult Human Achilles Tendon-An Anatomical Study," Annals of Anatomy,
Vol. 191, No. 6, 2009, pp. 586-593. doi:10.1016/j.aanat.2009.07.006

[48] H. Vyas and G. Krishnaswamy, "Images in Clinical Medicine. Quinolone-Associated Rupture of the Achilles' Tendon," New England Journal of Medicine, Vol. 357, No. 20, 2007, p. 2067. doi:10.1056/NEJMicm061227

[49] T. Movin, et al., "Pathology of the Achilles Tendon in Association with Ciprofloxacin Treatment," Foot \& Ankle International, Vol. 18, No. 5, 1997, pp. 297-299.

[50] C. B. Landersdorfer, et al., "Penetration of Moxifloxacin into Bone Evaluated by Monte Carlo Simulation," Antimicrobial Agents and Chemotherapy, Vol. 53, No. 5, 2009, pp. 2074-2081. doi:10.1128/AAC.01056-08 